\title{
Synthetic approaches to homogynolides A and B
}

\author{
A. Srikrishna, ${ }^{\star}$ S. Nagaraju, T. Jagadeeshwar Reddy and S. Venkateswarlu \\ Department of Organic Chemistry, Indian Institute of Science, Bangalore - 560 012, India.
}

\begin{abstract}
A four step, efficient and general methodology for the conversion of a cyclic ketone into the corresponding $\alpha$-spiro- $\beta$-methylene- $\gamma$-butyrolactone, the key structural feature present in tricyclic sesquiterpenes bakkanes, has been developed employing a regiospecific 5-exo dig radical cyclisation reaction as the key step. The methodology has been extended to the total synthesis of bakkanes including homogynolide-B and chiral homogynolide- $A$.
\end{abstract}

Bakkanes, biogenetically derived from eremophilanes, are an interesting class of tricyclic sesquiterpenes embodying an unusual $\alpha$-spiro- $\beta$-methylene- $\gamma$-butyrolactone fused to a hydrindane framework, ${ }^{1}$ and have been shown to possess cytotoxic and antifeedent properties. ${ }^{2}$ Homogynolides A and B, esters of 3- and 2-hydroxybakkenolides, were isolated ${ }^{3}$ from the neutral extracts of the plant Homogyne alpina (L.) CASs. along with bakkenolide-A. Despite their established biological properties and novel structural features, bakkanes have received only limited attention from synthetic chemists, ${ }^{4}$ and only Greene and his co-workers reported the total synthesis of homogynolides $\mathrm{A}$ and $\mathrm{B} .{ }^{5}$ With this background we have initiated a project on the total synthesis of bakkanes. First a four step, efficient and general methodology was developed ${ }^{6}$ for the conversion of a cyclic ketone into the corresponding $\alpha$-spiro- $\beta$ methylene- $\gamma$-butyrolactone employing a regiospecific 5-exo-dig radical cyclisation reaction as the key step.

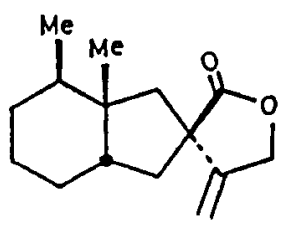

Bakkenolide-A (1)<smiles>C=C1COC(=O)C12CC1CC(OC(=O)/C(C)=C/C)CC(C)C1(C)C2</smiles>

Homogynolide-A (2)

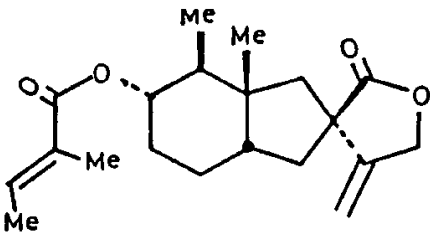

Homogynolide-B (3)

\section{Synthesis of $\alpha$-spiro- $\beta$-methylene- $\gamma$-butyrolactones:}

In contrast to the many procedures that exist for obtaining $\alpha$-methylene- $\gamma$-butyrolactones, there are very few general methods available for the synthesis of $\beta$-methylene- $\gamma$-butyrolactones. The last decade has witnessed a rapid growth in the use of free radical cyclisation reactions in organic synthesis. ${ }^{7}$ Based on the bromoacetal radical cyclisation reaction, originally developed by Stork and his co-workers, ${ }^{8}$ we have developed a four step, efficient method for the conversion of cyclic ketones into the $\alpha$-spiro- $\beta$ methylene- $\gamma$-butyrolactones. It was anticipated that the 5 -exo dig radical cyclisation of a suitable tertiary bromide will generate the requisite spiro centre, and bromoacetalisation reaction of an alkoxymethylenecyclic compound will generate the necessary bromoacetal. The sequence is depicted in the scheme 1 . Thus Wittig reaction on a cyclic ketone $\underline{4}$ with methoxymethylenetriphenylphosphorane followed by bromination of the resultant enol ether $\mathbf{5}$ with $\mathrm{N}$-bromosuccinimide (NBS) in the presence of prop-2-ynyl alcohol generated the requisite radical precursor, acetylenic bromoacetal 6 . The 5-exo dig radical cyclisation reaction of the bromoacetal 6 using in situ generated ${ }^{9}$ catalytic tri-n-butyltin hydride $\left({ }^{\circ} \mathrm{Bu}_{3} \mathrm{SnCl}\right.$ and $\left.\mathrm{NaCNBH}_{3}\right)$ in refluxing tert-butanol in the presence of a catalytic amount of azoisobutyronitrile (AIBN) furnished the spiroacetal 7 . Finally, sonochemically accelerated reaction of the spiroacetal $\underline{\mathbf{7}}$ with Jones reagent furnished the spirolactone $\underline{\mathbf{8}}$. Various cyclic ketones were transformed 


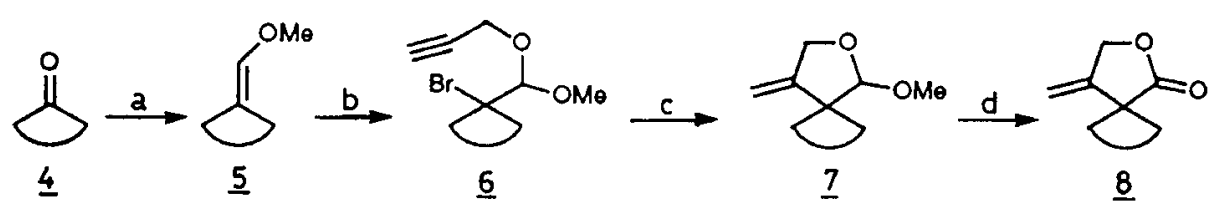

SCHEME 1: (a) $\mathrm{Ph}_{3} \mathrm{P}^{+}-\mathrm{CH}_{2} \mathrm{OMe}^{-C l}$, 'amy $\mathrm{O}^{+} \mathrm{K}, \mathrm{THF}, \mathrm{rt}, 2-5 \mathrm{~h}, 80-90 \%$; (b) $\mathrm{NBS}, \mathrm{HC} \equiv \mathrm{C}-\mathrm{CH}_{2} \mathrm{OH}$, $\mathrm{CH}_{2} \mathrm{Cl}_{2},-40^{\circ} \mathrm{C}, 0.5 \mathrm{~h}, 80-95 \%$; (c) " $\mathrm{Bu}_{3} \mathrm{SnCl}, \mathrm{NaCNBH}{ }_{3},{ }^{\mathrm{BuOH}}$, reflux, $1.5 \mathrm{~h}, 60-90 \%$; (d) Jones reagent, sonication, $\mathrm{Me}_{2} \mathrm{C}=0,5-15 \mathrm{~min}, 80-95 \%$.

into the corresponding $\alpha$-spiro- $\beta$-methylene- $\gamma$-butyrolactones employing this sequence including the hydrindanone $4 \mathrm{~h}$ into norbakkenolide-A $(\mathbf{8 h})$, and are listed in table 1. After successfully developing the methodology for the construction of the key spirolactone moiety of bakkanes, attention was focussed on the synthesis of homogynolides A (2) and B ( 3 ).

Table 1: Synthesis of $\alpha$-spiro- $\beta$-methylene- $\gamma$-butyrolactones

entry ketone 4

\section{Stereoselective Total Synthesis of Homogynolide B (3):}

Retrosynthetic analysis of homogynolide-B (3), based on the spirannulation described above, readily identified the ketospirolactone $\mathbf{9 a}$ (penultimate precursor in Greene's synthesis) and the ketoketal $\underline{10 a}$ as the key intermediates. ${ }^{10}$ Based on the Claisen rearrangement as the key step, Hagemann's ester $\underline{11}$ was opted as the starting material for the synthesis of the ketoketal $10 \mathrm{a}$. As a model study first Hagemann's ester $\underline{11}$ was successfully converted into the desmethyl compound $\underline{9 c}$, and the same methodology has been extended for the synthesis of the ketospirolactone 9a. Thus, monomethylation of 11 using sodium hydride and methyl iodide at $-50^{\circ} \mathrm{C}$ cleanly furnished the $\gamma$-alkylated product 12 with only trace amounts of $\alpha$-alkylated product. Ketalisation of $\underline{\mathbf{1 2}}$ using standard procedure gave the olefin isomerised ketal $\underline{13}$, which on reduction with $\mathrm{LiAlH}_{4}$ at low temperature generated the allyl alcohol $\underline{14}$ in a regiospecific manner. The one pot Claisen rearrangement of the allyl alcohol 14 with 2-methoxypropene in toluene in the presence of a catalytic amount of propionic acid furnished an inseparable epimeric mixture of the enone 15. Ozonolysis of the epimeric mixture $\underline{15}$ gave a 3:2 mixture of the epimeric diones $16 \mathrm{a}$, $\mathrm{mp} .90-92^{\circ} \mathrm{C}$ and $16 \mathrm{~b}$, which was separted by silica gel column chromatography. The generation of the two epimers in the Claisen rearrangement is of no consequence since the wrong isomer can be isomerised into right one at a later stage. The intramolecular aldol condensation of the dione 16a followed by the catalytic hydrogenation of the resultant enone 17 furnished the key intermediate, ketoketal $10 \mathrm{a}$. Finally radical mediated spirannulation transformed the ketoketal 10a into the ketospirolactone $9 \mathrm{a}$ in a highly 


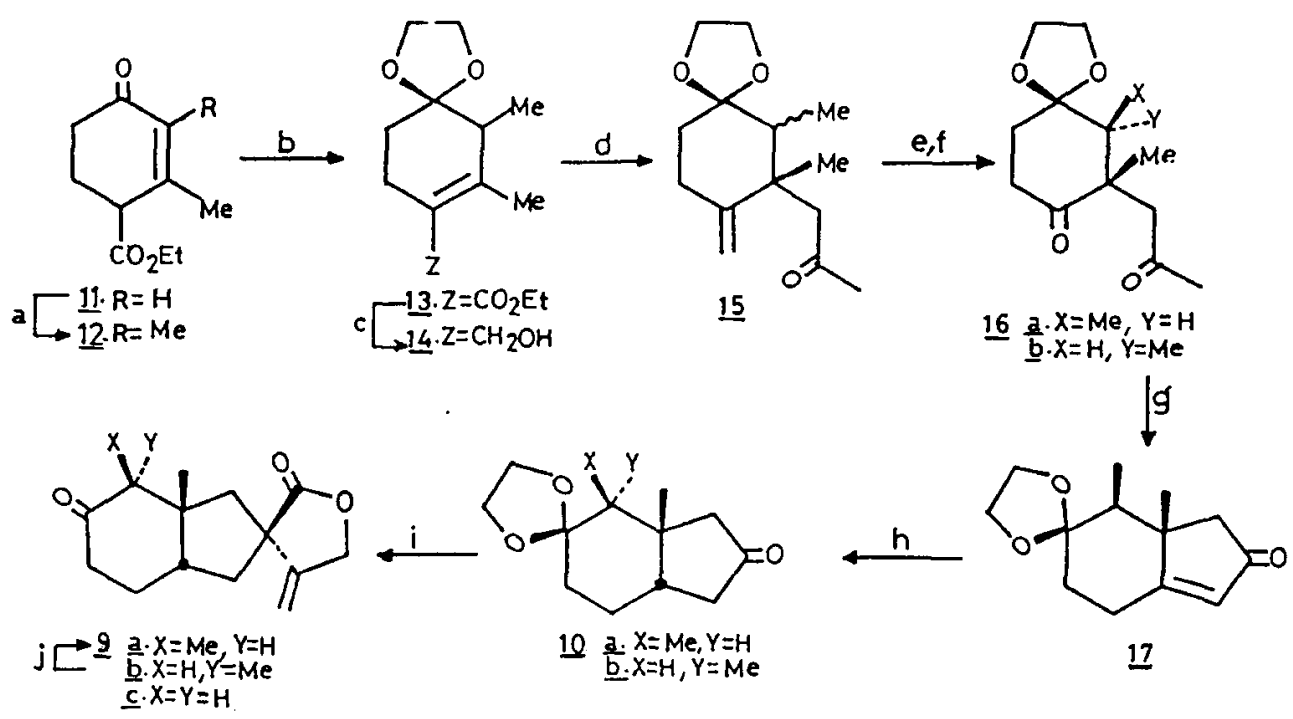

SCHEME 2: (a) NaH, Mel, THF, $-50^{\circ} \mathrm{C}$; (b) $\left(\mathrm{CH}_{2} \mathrm{OH}\right)_{2}, \mathrm{C}_{6} \mathrm{H}_{6}, \mathrm{p}-\mathrm{TSA}, \mathrm{reflux}$; (c) $\mathrm{LiAlH}_{4}, \mathrm{Et}_{2} \mathrm{O},-70^{\circ} \mathrm{C}$; (d) $\mathrm{CH}_{2}=\mathrm{C}(\mathrm{Me})-\mathrm{OMe}, \mathrm{PhMe}, 100^{\circ} \mathrm{C}, 10 \mathrm{~h} ; 190^{\circ} \mathrm{C}, 48 \mathrm{~h}$; (e) i. $\mathrm{O}_{3} \mathrm{O}_{2}, \mathrm{CH}_{2} \mathrm{Cl}_{2}-\mathrm{MeOH},-70^{\circ} \mathrm{C} ;$ ii. $\mathrm{Me}_{2} \mathrm{~S}$, $\mathrm{rt}$; (f) silica gel column chromatography; (g) $\mathrm{KOH}-\mathrm{MeOH}$, reflux; (h) $10 \% \mathrm{Pd} / \mathrm{C}-\mathrm{H}_{2}, \mathrm{EtOAC}, 1$ atm.; (i) same as scheme I; (j) $D B U, r t$.

stereoselective manner. In an analogous manner the epimeric dione $16 \mathbf{b}$ was converted into the ketospirolactone $9 \mathrm{~b}$ via the ketoketal $10 \mathrm{~b}$, which on epimerisation with DBU quantitatively furnished the ketospirolactone 9a. The high stereoselectivity in the construction of the spiro centre can be rationalised via the cyclisation of sterically preferred endo radical with the side chain occupying the exo position in the radical cyclisation reaction. Since Greene and co-workers have already converted ${ }^{\text {sb }}$ the ketospirolactone 9a into homogynolide-B (3), our synthesis of $9 \mathrm{a}$ constitutes a formal total synthesis of $\underline{3}$.

Currently this work is being extended to the total synthesis of chiral homogynolide-B employing the tartaric acid derived chiral diols for the chiral ketalisation of the methylated Hagemann's ester $\underline{\mathbf{1 2}}$.

\section{Synthetic Approaches to Chiral Homogynolide-A (2):}

After the successful completion of the total synthesis of homogynolide-B, we turned our attention towards the total synthesis of chiral homogynolide- $\mathrm{A}$. The spirolactone annulation methodology readily identified the ketospirolactone $\underline{18}$, a penultimate precursor in Greene's synthesis, and the ketoketal $\underline{19}$ as the key intermediates for the total synthesis of homogynolide-A (2). Visualising the isopropenyl group
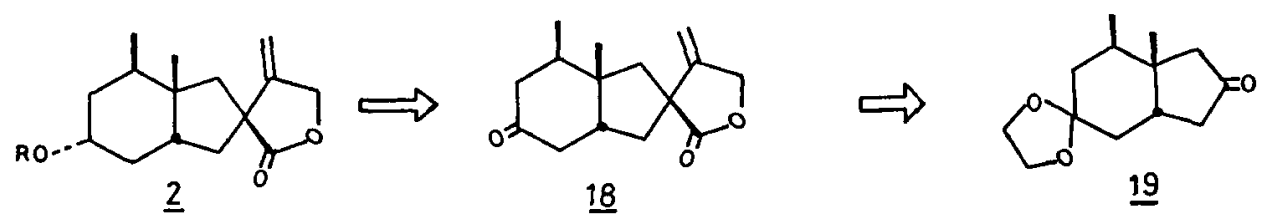

as equivalent to a hydroxy group, R-carvone (20) was chosen as the starting material. In our preliminary studies we have developed two different strategies, and converted R-carvone 20 into two optical antipodes of the ketoketal 19 (scheme 3). In the first method, the 1,4-conjugate addition of dimethyl copper lithium on carvone and trapping of the enolate with allyl bromide, cyclopentannulation via Wacker oxidation and degradation of the isopropenyl chain are the key reactions. Whereas in the second strategy, ortho ester Claisen rearrangement of carveol, intramolecular diazo ketone cyclopropanation, degradation of the isopropenyl chain, acid catalysed cyclopropane cleavage and 1,4-conjugate addition of dimethyl copper lithium are the key reactions. Finally radical mediated spirannulation transformed the ketoketal 19 into a mixture of the ketospirolactone $\underline{18}$ and its spiro epimer. Since the ketospirolactone $\underline{18}$ has already been converted $^{\text {sa }}$ into homogynolide-A (2) by Greene and co-workers, our synthesis of 18 constitutes a formal total synthesis of chiral homogynolide-A. 


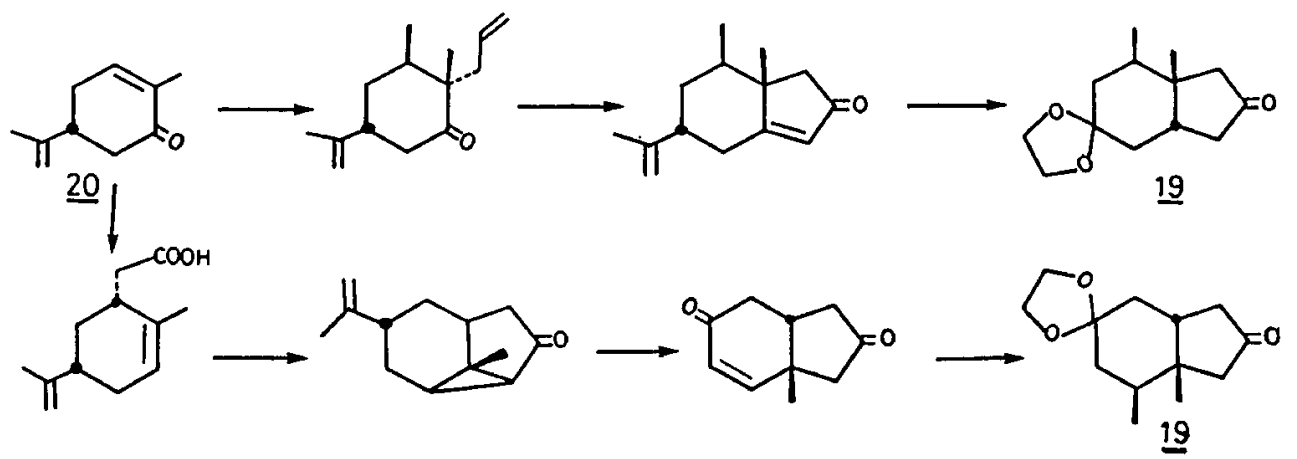

SCHEME 3

In conclusion, we have developed a methodology for the efficient conversion of a cyclic ketone into the corresponding $\alpha$-spiro- $\beta$-methylene- $\gamma$-butyrolactone, the key structural feature present in the tricyclic sesquiterpenoids bakkanes. The methodology has been extended to the total synthesis of homogynoloide-B and chiral homogynolide- $\mathrm{A}$.

Acknowledgements: The financial assistance from the Department of Science and Technology, New Delhi is gratefully acknowledged. Two of the authors, S.N. and T.J.R. thank the C.S.I.R. and U.G.C., New Delhi, respectively for the award of the research fellowships.

\section{REFERENCES}

1. N.H. Fischer, E.J. Olivier and H.D. Fischer, in Progress in the Chemistry of Organic Natural Products, W. Herz, H. Grisebach and G.W. Kirby Eds., Springer-Verlag, New York 1979, Vol. 38, Chapter 2 and references cited therein.

2. K. Kano, K. Hayashi and H. Mitsuhashi, Chem. Pharm. Bull., 30, 1198 (1982).

3. J. Harmatha, Z. Samek, M. Synackova, L. Novotny, V. Herout and F. Sorm, Collect. Czech. Chem. Commun., 41, 2047 (1976).

4. Bakkenolide-A: D.A. Evans, C.L. Sims and G.C. Andrews, J. Am. Chem. Soc., 99, 5453 (1977); A.E. Greene, J.-P. Depres, F. Coelho and T.J. Brocksom, J. Org. Chem., 50, 3943 (1985) and Tetrahedron Lett., 29, 5661 (1988).

5. (a) Homogynolide-A: B. Hartmann, A.M. Kanazawa, J.-P. Depres and A.E. Greene, Tetrahedron Lett., 32, 767 (1991) and 34, 3875 (1993); (b) Homogynolide-B: F. Coelho, J.-P. Depres, T.J. Brocksom and A.E. Greene, Tetrahedron Lett., 30, 565 (1989).

6. A. Srikrishna, S. Nagaraju and G.V.R. Sharma, J. Chem. Soc., Chem. Commun., 285 (1993); A. Srikrishna, T. Jagadeeshwar Reddy, S. Nagaraju and J.A. Sattigeri, Tetrahedron Lett., 35, 7841 (1994).

7. M. Ramaiah, Tetrahedron, 43, 3541 (1987); D.P. Curran, Synthesis, 417 and 489 (1988); C.P. Jasperse, D.P. Curran and T.L. Fevig, Chem. Rev., 91, 1237 (1991).

8. G. Stork, Bull. Chem. Soc. Jpn., 61, 149 (1988) and Bull. Soc. Chim. Fr., 127, 675 (1990).

9. G. Stork and P.M. Sher, J. Am. Chem. Soc., 108, 303 (1986); A. Srikrishna and G. Sundarababu, Tetrahedron, 46, 7901 (1990) and references cited therein.

10. A. Srikrishna, S. Nagaraju and S. Venkateswarlu, Tetrahedron Lett., 35, 429 (1994). 